\title{
Diversity of trypanorhynch metacestodes in teleost fishes from coral reefs off eastern Australia and New Caledonia
}

\author{
Ian Beveridge ${ }^{1, *}$, Rodney A. Bray ${ }^{2}$, Thomas H. Cribb ${ }^{3}$, and Jean-Lou Justine ${ }^{4}$ \\ 1 Veterinary Clinical Centre, University of Melbourne, Werribee, Victoria 3030, Australia \\ 2 Department of Life Sciences, Natural History Museum, Cromwell Road, London SW7 5BD, United Kingdom \\ 3 School of Biological Sciences, University of Queensland, Brisbane, Queensland 4072, Australia \\ 4 ISYEB, Institut de Systématique, Évolution, Biodiversité (UMR7205 CNRS, EPHE, MNHN, UPMC), Muséum National \\ d'Histoire Naturelle, CP 51, 55 rue Buffon, 75231 Paris Cedex 05, France
}

Received 31 August 2014, Accepted 31 October 2014, Published online November 182014

\begin{abstract}
Trypanorhynch metacestodes were examined from teleosts from coral reefs in eastern Australia and from New Caledonia. From over 12,000 fishes examined, 33 named species of trypanorhynchs were recovered as well as three species of tentacularioids which are described but not named. Host-parasite and parasite-host lists are provided, including more than 100 new host records. Lacistorhynchoid and tentacularioid taxa predominated with fewer otobothrioid and gymnorhynchoids. Five species, Callitetrarhynchus gracilis, Floriceps minacanthus, Pseudotobothrium dipsacum, Pseudolacistorhynchus heroniensis and Ps. shipleyi, were particularly common and exhibited low host specificity. Limited data suggested a higher diversity of larval trypanorhynchs in larger piscivorous fish families. Several fish families surveyed extensively (Blenniidae, Chaetodontidae, Gobiidae, Kyphosidae and Scaridae) yielded no trypanorhynch larvae. The overall similarity between the fauna of the Great Barrier Reef and New Caledonia was $45 \%$. Where available, information on the adult stages in elasmobranchs has been included.
\end{abstract}

Key words: Trypanorhyncha, Metacestodes, Great Barrier Reef, New Caledonia, Teleosts.

Résumé - Diversité des métacestodes de Trypanorhynques chez les téléostéens des récifs coralliens de l'est de l'Australie et de la Nouvelle-Calédonie. Les métacestodes de Trypanorhynques de téléostéens des récifs coralliens de l'est de l'Australie et de Nouvelle-Calédonie ont été examinés. À partir de plus de 12,000 poissons examinés, 33 espèces nommées de Trypanorhynques ont été collectées ainsi que trois espèces de Tentacularioidea qui sont décrites mais non nommées. Des listes hôtes-parasites et parasites-hôtes sont fournies, et incluent plus de 100 nouvelles mentions d'hôtes. Les taxa appartenant aux Lacistorhynchoidea et Tentacularioidea prédominaient et les Otobothrioidea et Gymnorhynchoidea étaient moins nombreux. Cinq espèces, Callitetrarhynchus gracilis, Floriceps minacanthus, Pseudotobothrium dipsacum, Pseudolacistorhynchus heroniensis et Ps. shipleyi étaient particulièrement fréquentes et montraient une faible spécificité d'hôte. Des données limitées suggèrent une plus grande diversité de Trypanorhynques larvaires dans les familles de poissons piscivores de grande taille. Plusieurs familles de poissons étudiées intensivement (Blenniidae, Chaetodontidae, Gobiidae, Kyphosidae et Scaridae) n'avaient pas de larves de Trypanorhynques. La similitude globale entre les faunes de la Grande Barrière de Corail et de la Nouvelle-Calédonie était de $45 \%$. Des informations sur les stades adultes chez des élasmobranches ont été incluses quand disponibles.

\section{Introduction}

The identification of significant threats to the coral reefs of the world [9, 17] has been partly responsible for focussing attention on the full diversity of reefs rather than simply on the diversity of fish and corals, the most obvious examples of reef diversity. The contributions of other groups of

\footnotetext{
*Corresponding author: ibeve@unimelb.edu.au
}

invertebrates to diversity on reefs have been largely overlooked in the past $[7,32]$. Part of this "hidden" invertebrate diversity includes the endoparasites of vertebrates.

In recent years, teleost fish occurring on coral reefs have been recognised as harbouring a particularly diverse array of parasites [20]. Studies to date have focussed either on specific parasite groups such as the Monogenea (e.g. [33]) or Digenea (e.g. [13]), or more recently have examined the diversity of all 
helminth parasites found in or on specific families of fish such as the Lethrinidae or Serranidae [21-23].

Teleosts found on coral reefs are commonly infected with the larval stages (plerocerci, merocerci or plerocercoids - for terminology see Chervy, 2002 [12]) of cestodes of the order Trypanorhyncha, the adults of which are found in the stomach or spiral valves of elasmobranchs. Larval stages occur most commonly in the body cavity but may also be found in the musculature or other sites such as the gill arches [27]. They constitute a significant component of parasite diversity but have frequently been overlooked because of taxonomic difficulties in identification [27]. However, unlike other orders of cestodes found in marine fish, the larval stages have scolex features, including the distinctive tentacular armature, which are identical to those found in the adult and which allow specific morphological identification. Although taxonomic studies of this group of parasites are frequent, ecological studies are few, and while systematic collecting has been undertaken in several parts of the world (Gulf of Mexico, Gulf of California, Java, Borneo, Australia and Hawaii), there are few published descriptions of the faunas encountered in these areas (see Jensen, 2009 [19] for Gulf of Mexico and Palm and Bray, 2014 [29] for Hawaii). Some species of trypanorhynchs (e.g. Grillotia (Christianella) minuta van Beneden, 1858; Gilquinia squali Fabricius, 1794) have also been used as biological tags in teleosts [25] because the larval stages are readily identifiable and because they are long-lived in the intermediate host. However, such ecological studies of these species are limited.

In this study, we examined the larval trypanorhynch cestode parasites of teleosts, and where applicable the corresponding adults in elasmobranchs, from the Great Barrier Reef (GBR) and compared them with those from similar reef environments in New Caledonia (NC). New Caledonia is separated from the GBR by about $1200 \mathrm{~km}$ of deep oceanic waters.

\section{Materials and methods}

\section{Great Barrier Reef (GBR)}

Teleosts and elasmobranchs were collected opportunistically between 1986 and 2010. The two main collecting sites were Heron Island in the southern Great Barrier Reef and Lizard Island in the Northern Barrier Reef. Small numbers of parasites were collected on reefs between these two sites (Mossman, Townsville) and in these instances, the nearest geographical feature on the coast was recorded rather than the specific reef near which the collection was made (Fig. 1).

Metacestodes were collected mainly from body cavities of teleosts, although in some instances they were sought in regions of the body such as the gill arches and musculature. Metacestodes were removed from surrounding cysts (in the case of plerocerci) and the eversion of tentacles was achieved either by shaking vigorously or by applying pressure under a coverslip. Cestodes were fixed in $70 \%$ ethanol or $10 \%$ formalin and were stained with Celestine blue or carmine (Palm, 2004) [27], dehydrated in ethanol, cleared in methyl salicylate and mounted in Canada balsam. All specimens were identified by IB and have been deposited in either the British Museum

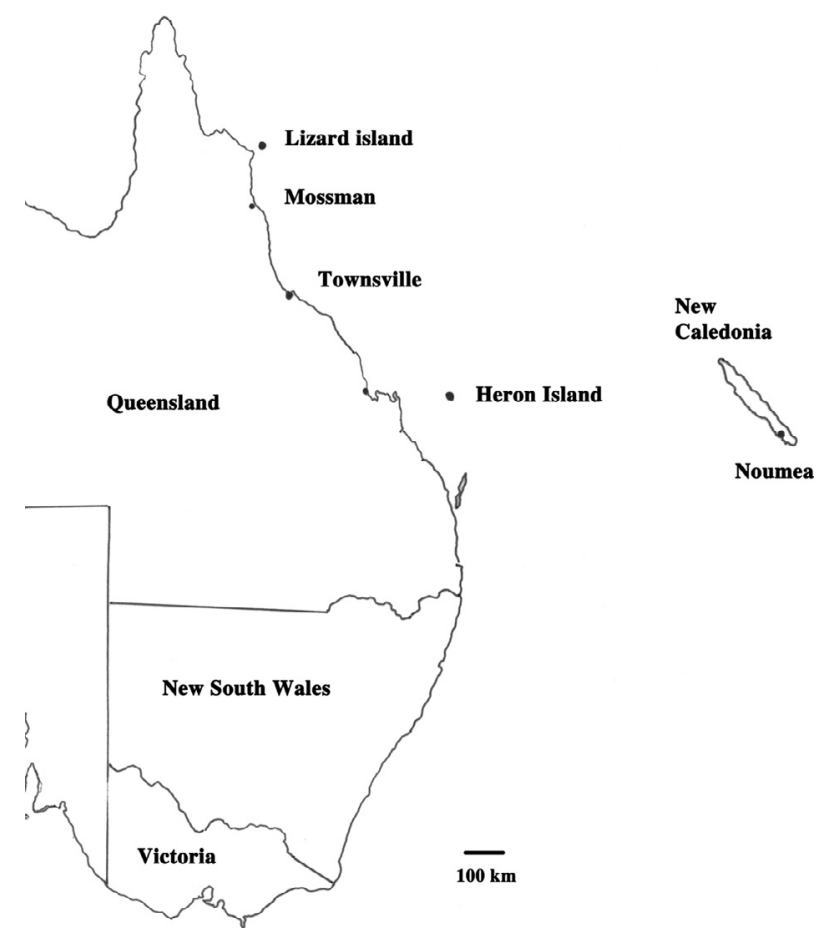

Figure 1. Collection localities off the east coast of Australia and New Caledonia.

(Natural History) (BMNH), the Queensland Museum, Brisbane (QM) or the South Australian Museum, Adelaide (SAM). Some of the records used in this compilation have been published previously in Beveridge \& Campbell, 1996, 2001 [1, 3], Beveridge et al., 2000, 2007 [4, 5], Campbell \& Beveridge, 1996 [8], Palm, 2004 [27], Palm \& Beveridge, 2002 [28] and Sakanari, 1989 [34].

Records of adults from elasmobranchs are included only for species in which larval stages have been identified in teleosts; these are based on both published data and specimens held in museum collections. Additional species of trypanorhynch cestodes from elasmobranchs have been found and their larval stages may be found in the future, but for the present study, these records have not been added.

\section{New Caledonia (NC)}

Fish were collected opportunistically between 2003 and 2009 generally by line fishing, occasionally by spear fishing and on occasions supplemented by fish obtained from a market. Collections were mainly off Nouméa (Fig. 1). All fish were measured, weighed and photographed. Methods for collection from several host families have been explained elsewhere [21-23]. Trypanorhynch plerocerci were opened and compressed between two slides or immersed in hot saline to evert tentacles. Plerocercoids found in the body cavity were also fixed under pressure to evert tentacles. Metacestodes were fixed in $70 \%$ ethanol or $10 \%$ formalin and were stained with Celestine blue or carmine [27], dehydrated in ethanol, cleared and mounted in Canada balsam. All specimens were identified by IB and have been deposited in the Muséum national 
d'Histoire naturelle, Paris (MNHN). Particular difficulties were encountered in the identification of tentaculariid cestodes from New Caledonia. Consequently, brief descriptions, some measurements and illustrations of each unidentified species encountered are included. Drawings were made with a drawing tube attached to an Olympus BH 2 microscope. Representative, rather than comprehensive, measurements were made with an ocular micrometer and are presented in micrometers.

In the parasite-host list (Table 1), authorities of cestodes are included and host species are listed in alphabetical order without authorities. In instances where both generic and specific names of cestodes have changed, synonyms have been included. In the host-parasite list (Table 2), fish hosts are arranged in orders, families and genera, but within each group, the order is alphabetical. Authorities of fish are indicated and the parasites are arranged in alphabetical order without authorities.

Authorities of hosts or parasites which are indicated in the lists are not repeated in the text. The systematic arrangement of trypanorhynch taxa follows Palm (2004) [27]. All host names were verified in FishBase [15]

\section{Results}

\section{Species found and other data}

Larval trypanorhynchs were recovered primarily from the body cavities of the teleosts examined (Figs. 2-7). Plerocerci were usually encountered attached to the mesentery enclosed within white envelopes (Fig. 2), although in some hosts melanisation of the cyst wall had occurred rendering the cysts brown (Fig. 3). Some brown or even black envelopes contained only remnants of plerocerci (Fig. 4). Plerocercoids of tentaculariids were found either in the body cavity or in the gastrointestinal lumen; the latter were not contained within a "cyst". Occasionally, plerocerci were found in the musculature and in the gill arches (Fig. 7), although there was no systematic search of such sites for plerocerci. Merocerci of Molicola horridus occurred in the livers of a limited number of species of teleosts, but the intensity of infection was high and the infections were readily observable at autopsy (Fig. 6).

Species of larval trypanorhynch cestodes found in both teleost (as larvae) and elasmobranch (as adult) hosts at sites along the GBR and off NC are shown in Tables 1 and 2.

From the GBR, the specimens examined were obtained from the dissection of more than 9000 fish, although not all were specifically examined for trypanorhynch cestodes. Likewise, from NC, approximately 3800 fish were examined but the body cavity was not examined in every fish, as explained by Justine et al. [21-23]. Consequently, prevalence data were available for some species only and abundance data were not available; for most species only presence-absence data were available (with one exception from Lizard Island).

No trypanorhynch metacestodes were found in the families Blenniidae $(n=215)$, Chaetodontidae $(n=1638)$, Gobiidae $(n=183)$, Kyphosidae $(n=30)$ and Scaridae $(n=147)$ from the GBR. Likewise, no metacestodes were found in the families Atherinidae $(n=13)$, Apogonidae $(n=19)$, Echeneidae $(n=10)$ and Haemulidae $(n=10)$ in NC. In addition, although the families Serranidae, Lethrinidae and Lutjanidae were frequently infected with trypanorhynch metacestodes, this pattern was not uniform across all species within these families and in NC, no trypanorhynch metacestodes were found in Epinephelus areolatus $(n=12)$, E. merra $(n=18)$, Lethrinus atkinsoni $(n=12), \quad$ L. nebulosus $(n=14), \quad$ Lutjanus fulviflamma $(n=10)$ and Lu. kasmira $(n=14)$.

Members of the Tentacularioidea differ from other trypanorhynch metacestodes as they are present as plerocercoids (= post-larvae) rather than plerocerci [14] and may be found in intestinal contents as well as in the viscera. In New Caledonia, tentacularioids were frequently found in smaller schooling fishes, often being the only trypanorhynchs encountered in these fishes.

In total, 33 named species were found (Tables 1 and 3) as well as three species of tentaculariid cestodes to which no current name could be applied. Lacistorhynchoid and tentacularioid trypanorhynchs dominated the fauna in terms of numbers of species recovered (Table 3), with the otobothrioid and gymnorhynchoid trypanorhynchs being less numerous.

Prevalence data were obtained from 182 fish from various families collected during a single collecting trip to Lizard Island. The prevalence of trypanorhynch larvae was: 4/6 $(77 \%)$ in scombrids, $5 / 7(71 \%)$ in lethrinids, $2 / 13(15 \%)$ in lutjanids, $8 / 9(89 \%)$ in serranids and $1 / 109(0.9 \%)$ in apogonids. Other fish families were represented by smaller numbers and were excluded.

\section{Tentacularioid metacestodes of uncertain identity}

Superfamily Tentacularioidea Poche, 1926

Family Tentaculariidae Poche, 1926

\section{Nybelinia sp. A (Fig. 8)}

Material examined: plerocercoids from Herklotsichthys quadrimaculatus (Rüppell, 1937), New Caledonia, MNHN JNC2669C1, 2671A1.

Scolex length 1200 , pars bothrialis 580, pars vaginalis 520; bulbs ovoid, bulb length 250; velum 160; metabasal hooks: length 15 , base 10 .

Remarks

This species is similar to $N$. queenslandensis, but all measurements including those of the hooks are substantially smaller. In addition, the shape of the hooks differs (Fig. 8). The hook shape aligns the species with $N$. lingualis (Cuvier, 1817), N. bisulcata (Linton, 1889), N. anthicosum Heinz \& Dailey, 1974 and N. hemipristis Palm \& Beveridge, 2002, but $N$. lingualis and $N$. bisulcata differ in having much larger scoleces (2025-2700 and 2500, respectively) and bulbs (365425 and 450-505, respectively) while the latter two species have much larger hooks (25-40). Consequently, these 
Table 1. Parasite-host list. Species of trypanorhynch cestodes collected from teleosts and elasmobranchs on the Great Barrier Reef, Australia and from New Caledonia. Authorities of

\begin{tabular}{|c|c|c|c|}
\hline & Great Barrier Reef & New Caledonia & \\
\hline \multicolumn{4}{|l|}{ GYMNORHYNCHOIDEA } \\
\hline \multicolumn{4}{|l|}{ Molicola horridus (Goodsir, 1841) } \\
\hline \multicolumn{4}{|l|}{ Larval } \\
\hline Diodon hystrix & $\begin{array}{c}\mathrm{H}^{\mathrm{A}} \mathrm{QM}^{\mathrm{C}} \mathrm{G} 206954 \\
\mathrm{SAM}^{\mathrm{E}} 44079\end{array}$ & Diodon hystrix & MNHN $^{\mathrm{D}} \mathrm{JNC}$ 2977D1, 3199C \\
\hline Diodon liturosus* & $\mathrm{L}^{\mathrm{B}} \mathrm{QM} \mathrm{G} 232552$ & & \\
\hline \multicolumn{4}{|c|}{ Pterobothrium lintoni MacCallum, 1916} \\
\hline \multicolumn{4}{|c|}{ Larval } \\
\hline Choerodon venustus & H SAM 40480 & & \\
\hline \multicolumn{4}{|l|}{ Pterobothrium acanthotruncatum } \\
\hline \multicolumn{4}{|l|}{ Escalante \& Carvajal, 1984} \\
\hline \multicolumn{4}{|l|}{ Larval } \\
\hline Plectropomus maculatus* & H QM G217640 & & \\
\hline Scomberomorus commerson & H, L QM G217628 & & \\
\hline \multicolumn{4}{|l|}{ Adult } \\
\hline Pristis zijsron* & $\mathrm{Tv}^{\mathrm{G}}$ SAM 35749 & & \\
\hline \multicolumn{4}{|c|}{ Pterobothrium australiense Campbell \& } \\
\hline \multicolumn{4}{|c|}{ Beveridge, 1996} \\
\hline \multicolumn{4}{|l|}{ Larval } \\
\hline Halichoeres trimaculatus* & H QM G217629 & & \\
\hline \multicolumn{4}{|l|}{ Adult } \\
\hline Pristis zijsron & Tv SAM 23898 & & \\
\hline \multicolumn{4}{|c|}{ Pterobothrium pearsoni (Southwell, 1929) } \\
\hline \multicolumn{4}{|c|}{ Larval } \\
\hline Sphyraena jello* & L QM G233646 & & \\
\hline \multicolumn{4}{|c|}{ LACISTORHYNCHOIDEA } \\
\hline \multicolumn{4}{|c|}{ Bombycirhynchus sphaerenaicum (Pintner, 1930) } \\
\hline \multicolumn{4}{|c|}{ Larval } \\
\hline Sphyraena jello* & L QM G233583 & & \\
\hline \multicolumn{4}{|c|}{ Callitetrarhynchus gracilis (Rudolphi, 1819) } \\
\hline \multicolumn{4}{|c|}{ Larval } \\
\hline Abudefduf whitleyi* & H QM G212162 & Atule mate* & $\begin{array}{l}\text { MNHN JNC2814T, 2963, 2964, } \\
2965,3371\end{array}$ \\
\hline Apogon poecilopterus* & H QM G217587 & Carangoides fulvoguttatus* & MNHN JNC463C \\
\hline Caesio cuning* & H QM G217593 & Caranx papuensis* & MNHN JNC1189E \\
\hline Cephalopholis miniata & H QM G232625 & Cephalopholis boenak & {$[20]^{\mathrm{J}}$} \\
\hline Cephalopholis cyanostigma* & H, L QM G217575 & Cephalopholis spiloparaea* & MNHN JNC2624 \\
\hline Choerodon cyanodus* & Н ВМ 1980.7.10.148-9 & Chirocentrus dorab & MNHN JNC3220 \\
\hline Cromileptes altivelis* & H QM G217592 & Epinephelus chlorostigma & MNHN JNC2446C \\
\hline Johnius borneensis* & H QM G217602 & Epinephelus fasciatus* & MNHN JNC1256A, 2625, 3039 \\
\hline Lotella rhacina* & H QM G217574 & Epinephelus retouti* & MNHN JNC3083 \\
\hline Lutjanus carponotatus* & L QM G233588 & Epinephelus rivulatus & MNHN JNC2606C \\
\hline
\end{tabular}




\begin{tabular}{|c|c|c|c|}
\hline & Great Barrier Reef & New Caledonia & \\
\hline Naso vlamingii* & H QM G217598 & Lethrinus miniatus* & MNHN JNC2113A \\
\hline Ostorhinchus fasciatus* & H QM G217486 & Lutjanus vitta & {$[22]^{\mathrm{J}}$} \\
\hline Plectropomus maculatus* & H QM G217641 & Megalaspis cordyla & MNHN JNC1186, 1188 \\
\hline Polynemus heptadactyla* & H QM G217591 & Nemipterus furcosus & MNHN JNC2596 \\
\hline Pomatomus saltatrix & H QM G217583 & Scomberomorus commerson & MNHN JNC435 \\
\hline Scomberomorus commerson & H, L QM G212163 & Triodon macropterus $*$ & MNHN JNC2984 \\
\hline Scomberomorus queenslandicus* & H QM G217588 & Variola louti & {$[20]^{\mathrm{J}}$} \\
\hline Sphyraena obtusata* & H QM G217590 & & \\
\hline \multicolumn{4}{|l|}{ Adult } \\
\hline Carcharhinus melanopterus & H QM G217581 & Carcharhinus leucas & MNHN JNC2856 \\
\hline Carcharhinus amblyrhynchoides & $\mathrm{Si}^{\mathrm{H}} \mathrm{SAM} 24941$ & & \\
\hline \multicolumn{4}{|c|}{ Callitetrarhynchus speciosus (Linton, 1897) } \\
\hline Larval & & Cymbacephalus beauforti* & MNHN JNC1833 \\
\hline \multicolumn{4}{|c|}{ Dasyrhynchus basipunctatus (Carvajal, Campbell \& } \\
\hline \multicolumn{4}{|c|}{ Cornford, 1976) } \\
\hline \multirow[t]{6}{*}{ Fistularia commersonii* } & H QM G232633 & Abalistes filamentosus* & MNHN JNC2193 \\
\hline & & Abalistes stellatus & MNHN JNC2163, 2926, 2914 \\
\hline & & Diodon hystrix & MNHN JNC2977 \\
\hline & & Lagocephalus sceleratus* & MNHN JNC2942 \\
\hline & & Pseudobalistes fuscus* & MNHN JNC1680E, 2164, 2940 \\
\hline & & Triodon macropterus* & MNHN JNC2989 \\
\hline \multicolumn{4}{|l|}{ Adult } \\
\hline Carcharhinus brachyurus & L QM G232540 & Carcharhinus amblyrhynchos & MNHN JNC435, 1111 \\
\hline Carcharhinus melanopterus* & H QM G232634 & Carcharhinus plumbeus* & MNHN JNC442 \\
\hline \multicolumn{4}{|c|}{ Diesingium cf lomentaceum (Diesing, 1850) } \\
\hline \multicolumn{4}{|c|}{ Larval } \\
\hline & & Carangoides fulvoguttatus* & MNHN JNC3169 \\
\hline & & Epinephelus chlorostigma* & MNHN JNC3142 \\
\hline \multirow{2}{*}{\multicolumn{4}{|c|}{$\begin{array}{l}\text { Floriceps minacanthus Campbell \& Beveridge, } 1987 \\
\text { Larval }\end{array}$}} \\
\hline & & & \\
\hline Cephalopholis boenak* & H QM G212151-3 & Cephalopholis miniata & MNHN JNC2627 \\
\hline Cephalopholis cyanostigma* & L QM G233613 & Cephalopholis sonnerati* & MNHN JNC2934, 2935, 2936, 3029 \\
\hline Cephalopholis miniata & H QM G217615 & Cephalopholis urodeta & {$[20]^{\mathrm{J}}$} \\
\hline Epinephelus quoуanus & H SAM 44083 & Epinephelus coioides & MNHN JNC3257 \\
\hline Euthynnus affinis & H QM G217612, 7 & Epinephelus cyanopodus & MNHN JNC1998 \\
\hline Euthynnus alletteratus* & H SAM 44082 & Epinephelus maculatus* & MNHN JNC2937, 3061, 3062, 3066 \\
\hline Grammatorcynus bicarinatus* & H, L QM G217613 & Lethrinus miniatus* & MNHN JNC2706A \\
\hline Lethrinus miniatus* & H QM G233554 & Nemipterus furcosus & MNHN JNC3019 \\
\hline Plectropomus areolatus* & L QM G233626 & Plectropomus leopardus & MNHN JNC2585A \\
\hline Plectropomus leopardus & H, L QM G217611, SAM 32139 & Plectropomus laevis & MNHN JNC1887 \\
\hline Sphyraena flavicauda* & H QM G217616 & Sphyraena putnamae* & MNHN JNC3035 \\
\hline Sphyraena jello* & L QM G233610 & Tylosurus crocodilus* & MNHN JNC1262C, 1263A \\
\hline Tylosurus crocodilus* & H QM G217614 & Variola louti & MNHN JNC1859B, 3037 \\
\hline
\end{tabular}




\begin{tabular}{|c|c|c|c|}
\hline & Great Barrier Reef & New Caledonia & \\
\hline \multicolumn{4}{|l|}{ Adult } \\
\hline \multirow[t]{2}{*}{ Carcharhinus amboinensis } & StL $^{\mathrm{I}} \mathrm{SAM} 22652$ & Carcharhinus leucas* & MNHN \\
\hline & & Triaenodon obesus* & MNHN \\
\hline \multicolumn{4}{|l|}{ Floriceps saccatus (Cuvier, 1817) } \\
\hline \multicolumn{4}{|l|}{ Larval } \\
\hline Diodon hystrix & H SAM 44081 & Caranx papuensis* & MNHN JNC3209 \\
\hline Diodon liturosus* & L QM G232554 & Diodon hystrix & MNHN JNC2343, 2977, 3199 \\
\hline \multicolumn{4}{|l|}{ Grillotiella exile (Linton, 1909) } \\
\hline \multicolumn{4}{|l|}{ Larval } \\
\hline Scomberomorus commerson & L QM G233632 & & \\
\hline Adult & & Galeocerdo cuvier & MNHN JNC1414 \\
\hline \multicolumn{4}{|c|}{ Microbothriorhynchus coelorhynchi Yamaguti, 1952} \\
\hline Larval & & Conger cinereus* & MNHN JNC2993 \\
\hline \multicolumn{4}{|c|}{$\begin{array}{l}\text { Pseudogilquinia microbothria } \text { (MacCallum, 1917) } \\
(=\text { Ps. magna; = Dasyrhynchus magnus) }\end{array}$} \\
\hline \\
\hline Lethrinus atkinsoni* & L QM G233653 & Lethrinus miniatus* & MNHN JNC2113 B1, 2158C \\
\hline Lethrinus nebulosus* & L QM G233654 & & \\
\hline \multicolumn{4}{|c|}{ Pseudogilquinia pillersi (Southwell, 1929) } \\
\hline \multicolumn{4}{|c|}{ Larval } \\
\hline Lethrinus atkinsoni & $\mathrm{H} \mathrm{BM}^{\mathrm{F}}$ 2004.3.18.98-99 & Epinephelus coioides & MNHN JNC1535, 3140, 3265B \\
\hline Lethrinus miniatus & H ВM 2004.3.18.97 & Plectropomus laevis & MNHN JNC1865, 1887 \\
\hline Lethrinus nebulosus & L QM G233653 & Epinephelus malabaricus & MNHN JNC1536 \\
\hline \multicolumn{4}{|c|}{ Pseudolacistorhynchus heroniensis (Sakanari, 1989) } \\
\hline \multicolumn{4}{|c|}{ Larval } \\
\hline Cephalopholis miniata* & H QM G212146 & Abalistes filamentosus* & MNHN JNC2724 \\
\hline Epinephelus fasciatus & H QM G217518, SAM 17418 & Abalistes stellatus* & MNHN JNC2163, 2914, 2926 \\
\hline Epinephelus ongus* & H QM G214949 & Cephalopholis boenak & MNHN JNC2889, 2890, 3205 \\
\hline Epinephelus quoyanus & H QM G212157, SAM 28629 & Cephalopholis sonnerati* & MNHN JNC2934 \\
\hline Lethrinus miniatus* & H QM G212154 & Gymnocranius grandoculis* & MNHN JNC1726 \\
\hline Lethrinus nebulosus & $\mathrm{H}[24]^{\mathrm{J}}$ & Epinephelus chlorostigma & MNHN JNC2446C, 3141 \\
\hline \multirow[t]{10}{*}{ Plectropomus leopardus } & H QM G212158, SAM 28681 & Epinephelus coioides* ${ }^{*}$ & MNHN JNC3257 \\
\hline & & Epinephelus cyanopodus & {$[20]^{J}$} \\
\hline & & Epinephelus fasciatus & $\begin{array}{l}\text { MNHN JNC1636A, 1758, } \\
1791,1792,3039\end{array}$ \\
\hline & & Epinephelus howlandi* & MNHN JNC2768 \\
\hline & & Epinephelus polyphekadion & MNHN JNC1915C, 3036 \\
\hline & & Epinephelus rivulatus & MNHN JNC1545C \\
\hline & & Lethrinus miniatus* & MNHN JNC2161C \\
\hline & & Lutjanus vitta* & {$[22]^{J}$} \\
\hline & & Plectropomus leopardus & MNHN JNC3279 \\
\hline & & Pseudobalistes fuscus* & MNHN JNC2164, 2940B \\
\hline Adult & & Stegostoma fasciatum & MNHN JNC1529 \\
\hline
\end{tabular}


Pseudolacistorhynchus shipleyi (Southwell, 1929)

(= Grillotia overstreeti Sakanari, 1989)

Larval

Cephalopholis boenak*

Cephalopholis cyanostigma*

Choerodon cyanodus

Choerodon fasciatus*

Epinephelus ongus*

Lotella rhacina*

Rhinecanthus aculeatus*

Sufflamen fraenatus*

OTOBOTHRIOIDEA

Otobothrium alexanderi Palm, 2004

Larval

Tylosurus crocodilus

Otobothrium parvum Beveridge \& Justine, 2007 Larval

Adult

Otobothrium penetrans Linton, 1907

Larval $^{\dagger}$

Proemotobothrium southwelli Beveridge \&

Campbell, 2001

Larval

Johnius borneensis

Pseudotobothrium dipsacum (Linton, 1897)

Larval

Abalistes stellatus

Cephalopholis cyanostigma

Cheilinus trilobatus

Epinephelus coioides

Lethrinus obsoletus

Lutjanus gibbus

Naso vlamingii

Plectropomus leopardus

Plectropomus maculatus

Pseudocaranx dentex

Rhinecanthus aculeatus

Rhinecanthus rectangulus
H QM G232626

H, L QM G214957

H SAM 17416, QM G212160

H QM G217519

H QM G212161

H QM G214995

L QM G232542

H QM G217520

L QM G232555

H QM G217939

H QM G217928-32

H QM G214959

L QM G233555

Tv SAM 31342

H QM G233888

L QM GL 10508

H QM G214960

H, L QM G217936

H QM G206964

H QM G214961

L QM G232590

H QM G217934
Tylosurus crocodilus

Epinephelus maculatus

Lethrinus rubrioperculatus*

Carcharhinus amblyrhynchos

Triaenodon obesus

Tylosurus crocodilus

Cephalopholis sonnerati*

Cephalopholis urodeta

Einephelus polyphekadion*

Epinephelus ongus*

MNHN JNC1968

MNHN JNC1405

MNHN JNC1635A

MNHN JNC1111

MNHN JNC2109

MNHN JNC1968

Abalistes filamentosus*

Abalistes stellatus

Cephalopholis miniata*

Cephalopholis sonnerati*

Cephalopholis urodeta

Cymbacephalus beauforti*

Epinephelus coioides

Epinephelus fasciatus*

Epinephelus malabaricus

Epinephelus retouti*

Plectropomus laevis*

Plectropomus leopardus

Pseudobalistes fuscus*

Variola louti

MNHN JNC2179
MNHN JNC2724

MNHN JNC2914

MNHN JNC2627

MNHN JNC1616, 2934-6

MNHN JNC2750

MNHN JNC1833A

MNHN JNC1535, 3257

MNHN JNC1791, 3039

$[20]^{\mathrm{J}}$

MNHN JNC1865, 1887

MNHN JNC2126

MNHN JNC2927, 2940

MNHN JNC1629, 1662, 1756-7, 
Symbothriorhynchus tigaminacanthus

Palm, 2004

Larval

Adult

TENTACULARIOIDEA

Hepatoxylon trichiuri

Larval

Diodon hystrix*

Adult ${ }^{\dagger}$

Heteronybelinia estigmena (Dollfus, 1960)

Larval

Sarda australis

H QM G218042-6

Adult

Carcharhinus sp.

Heteronybelinia sp. C

Myxonybelinia southwelli (Palm \& Walter, 1999)

Larval

Choerodon venustus

Adult

Nybelinia aequidentata Shipley \& Hornell, 1906 Larval

Nybelinia basimegacantha Carvajal, Campbell \&

Cornford, 1976

Larval

Parupeneus bifasciatus*

Nybelinia goreensis Dollfus, 1960

Larval

Great Barrier Reef

New Caledonia

H QM G227128

Nemipterus furcosus*

Saurida undosquamis*

Sphyrna lewini

Diodon hystrix*

Tetrapterus angustirostris*

Thunnus obesus*

Prionace glauca*

Atule mate*

Herklotsichthys quadrimaculatus*

Selar crumenophthalmus

Sphyraena putnamae*

Trichiurus lepturus*

Qld SAM 18322

Carcharhinus brevipinna

Sufflamen fraenatus

Stegostoma fasciatum

Dendrochirus zebra*

Neoniphon sammara*

Parupeneus multifasciatus

Lethrinus genivittatus*

Lethrinus rubrioperculatus*

Nemipterus furcosus

Parupeneus barberinus*

Parupeneus multifasciatus*
MNHN JNC2586, 2610

MNHN JNC2079

MNHN JNC1628

MNHN JNC2977, 3199D

MNHN JNC1399

MNHN JNC1398

MNHN JNC1217

MNHN JNC2963-5

MNHN JNC2669B, 2673, 2943, 2949

MNHN JNC3043-4, 3126

MNHN JNC3035

MNHN JNC3045-6, 3048

MNHN JNC3138

MNHN JNC3034

MNHN JNC1529

QM G218031

MNHN JNC2552

MNHN JNC2111

MNHN JNC2033

MNHN JNC1148

MNHN JNC2612

MNHN JNC1838B

MNHN JNC2112 
Nybelinia indica Chandra, 1986

(= Nybelinia scoliodoni Vijayalakshmi,

Vijayalakshmi \& Gangadharam, 1996)

Larval

Diodon hystrix
H QM G218034-41

H SAM 17646

H QM G232539

L QM G2336644

H, L QM G217521-31

H QM G218109
Carcharhinus melanopterus

Larval

Johnius borneensis

Nybelinia sp. A

Larval

Nybelinia sp. B

Larval

MNHN JNC2669C

Parupeneus multifasciatus

MNHN JNC3194

MNHN JNC2977F

MNHN JNC2982

MNHN JNC2921

MNHN JNC2288, 2611, 3016

MNHN JNC2109B1

MNHN JNC3011-2

New host records.

Heron Island, Great Barrier Reef.

B Lizard Island, Great Barrier Reef.

${ }^{\mathrm{C}}$ Queensland Museum, Brisbane.

D Muséum national d'Histoire naturelle, Paris.

${ }^{\mathrm{E}}$ South Australian Museum, Adelaide.

F British Museum, Natural History, London.

G Townsville, Queensland.

H Snapper Island, Mossman.

${ }^{\text {I }}$ St Lawrence, Queensland.

${ }^{J}$ Published report not supported by museum specimen.
MNHN JNC2172C 
Table 2. Species of trypanorhynch cestodes collected from teleosts on the Great Barrier Reef, Australia and from New Caledonia. Authorities of fish are included and cestodes are listed in alphabetical order without authorities. GBR: Great Barrier Reef; NC: New Caledonia.

\begin{tabular}{|c|c|c|c|c|}
\hline Order & Family & Host species & Parasites & Location \\
\hline Anguilliformes & Congridae & Conger cinereus Rüppell, 1830 & Microbothriorhynchus coelorhynchi & $\mathrm{NC}$ \\
\hline Aulopiformes & Synodontidae & Saurida undosquamis (Richardson, 1848) & Symbothriorhynchus tigaminacanthus & $\mathrm{NC}$ \\
\hline \multirow[t]{3}{*}{ Beloniformes } & Belonidae & Tylosurus crocodilus (Péron \& Lesueur, 1821) & Floriceps minacanthus & GBR, NC \\
\hline & & & Otobothrium alexanderi & GBR, NC \\
\hline & & & Otobothrium penetrans & $\mathrm{NC}$ \\
\hline Beryciformes & Holocentridae & Neoniphon sammara (Forsskål, 1775) & Nybelinia basimegacantha & $\mathrm{NC}$ \\
\hline \multirow[t]{3}{*}{ Clupeiformes } & Chirocentridae & Chirocentrus dorab (Forsskål, 1775) & Callitetrarhynchus gracilis & $\mathrm{NC}$ \\
\hline & Clupeidae & Herklotsichthys quadrimaculatus (Rüppell, 1837) & Heteronybelinia estigmena & $\mathrm{NC}$ \\
\hline & & & Nybelinia sp. A & $\mathrm{NC}$ \\
\hline \multirow[t]{2}{*}{ Gadiformes } & Moridae & Lotella rhacina (Forster, 1801) & Callitetrarhynchus gracilis & GBR \\
\hline & & & Pseudolacistorhynchus shipleyi & GBR \\
\hline \multirow[t]{45}{*}{ Perciformes } & Acanthuridae & Naso vlamingii (Valenciennes, 1835) & Callitetrarhynchus gracilis & GBR \\
\hline & & & Pseudotobothrium dipsacum & GBR \\
\hline & Apogonidae & Apogon poecilopterus Cuvier, 1828 & Callitetrarhynchus gracilis & GBR \\
\hline & & Ostorhinchus cookii (Macleay, 1881) & Nybelinia queenslandensis & GR \\
\hline & & Ostorhinchus fasciatus (White, 1790) & Callitetrarhynchus gracilis & GBR \\
\hline & & Ostorhinchus properuptus (Whitley, 1964) & Nybelinia queenslandensis & GBR \\
\hline & Carangidae & Atule mate (Cuvier, 1833) & Callitetrarhynchus gracilis & $\mathrm{NC}$ \\
\hline & & & Heteronybelinia estigmena & $\mathrm{NC}$ \\
\hline & & Carangoides fulvoguttatus (Forsskål, 1775) & Callitetrarhynchus gracilis & $\mathrm{NC}$ \\
\hline & & & Diesingium cf lomentaceum & $\mathrm{NC}$ \\
\hline & & Carangoides sexfasciatus Quoy \& Gaimard, 1825 & Nybelinia indica & $\mathrm{NC}$ \\
\hline & & Caranx papuensis Alleyne \& Macleay, 1877 & Callitetrarhynchus gracilis & $\mathrm{NC}$ \\
\hline & & & Floriceps saccatus & $\mathrm{NC}$ \\
\hline & & Megalaspis cordyla (Linnaeus, 1758) & Callitetrarhynchus gracilis & $\mathrm{NC}$ \\
\hline & & Pseudocaranx dentex (Bloch \& Schneider, 1801) & Pseudotobothrium dipsacum & GBR \\
\hline & & Selar crumenophthalmus (Bloch, 1793) & Heteronybelinia estigmena & $\mathrm{NC}$ \\
\hline & & Tetrapterus angustirostris Tanka, 1915 & Hepatoxylon trichiuri & $\mathrm{NC}$ \\
\hline & Labridae & Cheilinus trilobatus (Lacépède, 1801) & Pseudotobothrium dipsacum & GBR \\
\hline & & Halichoeres trimaculatus (Quoy \& Gaimard, 1834) & Pterobothrium australiense & GBR \\
\hline & & Choerodon cyanodus (Richardson, 1843) & Callitetrarhynchus gracilis & GBR \\
\hline & & & Pseudolacistorhynchus shipleyi & GBR \\
\hline & & Choerodon fasciatus (Günther, 1867) & Pseudolacistorhynchus shipleyi & GBR \\
\hline & & Choerodon venustus (De Vis, 1884) & Myxonybelinia southwelli & GBR \\
\hline & & & Pterobothrium lintoni & GBR \\
\hline & Leiognathidae & Leiognathus fasciatus (Lacépède, 1803) & Nybelinia indica & $\mathrm{NC}$ \\
\hline & Lethrinidae & Lethrinus atkinsoni Seale, 1910 & Pseudogilquinia microbothria & GBR \\
\hline & & & Pseudogilquinia pillersi & GBR \\
\hline & & Lethrinus genivittatus Valenciennes, 1830 & Nybelinia goreensis & $\mathrm{NC}$ \\
\hline & & Lethrinus miniatus (Forster, 1801) & Callitetrarhynchus gracilis & $\mathrm{NC}$ \\
\hline & & & Floriceps minacanthus & GBR, NC \\
\hline & & & Pseudolacistorhynchus heroniensis & GBR, NC \\
\hline & & & Pseudogilquinia microbothria & $\mathrm{NC}$ \\
\hline & & & Pseudogilquinia pillersi & GBR \\
\hline & & Lethrinus nebulosus (Forsskål, 1775) & Pseudogilquinia microbothria & GBR \\
\hline & & & Pseudogilquinia pillersi & GBR \\
\hline & & & Pseudolacistorhynchus heroniensis & GBR \\
\hline & & Lethrinus obsoletus (Forsskål, 1775) & Pseudotobothrium dipsacum & GBR \\
\hline & & Lethrinus rubrioperculatus Sato, 1978 & Nybelinia goreensis & $\mathrm{NC}$ \\
\hline & & & Otobothrium parvum & $\mathrm{NC}$ \\
\hline & & Gymnocranius grandoculis (Valenciennes, 1830) & Pseudolacistorhynchus heroniensis & $\mathrm{NC}$ \\
\hline & Lutjanidae & Caesio cuning (Bloch, 1791) & Callitetrarhynchus gracilis & GBR \\
\hline & & Lutjanus carponotatus (Richardson, 1842) & Callitetrarhynchus gracilis & GBR \\
\hline & & Lutjanus gibbus (Forsskål, 1775) & Pseudotobothrium dipsacum & GBR \\
\hline & & Lutjanus vitta (Quoy \& Gaimard, 1824) & Callitetrarhynchus gracilis & $\mathrm{NC}$ \\
\hline & & & Pseudolacistorhynchus heroniensis & $\mathrm{NC}$ \\
\hline
\end{tabular}


Table 2. (continued)

\begin{tabular}{|c|c|c|c|c|}
\hline Order & Family & Host species & Parasites & Location \\
\hline & \multirow[t]{5}{*}{ Mullidae } & Parupeneus barberinus (Lacépède, 1801) & Nybelinia goreensis & $\mathrm{NC}$ \\
\hline & & Parupeneus bifasciatus (Lacépède, 1801) & Nybelinia basimegacantha & GBR \\
\hline & & Parupeneus multifasciatus (Quoy \& Gaimard, 1825) & Nybelinia basimegacantha & $\mathrm{NC}$ \\
\hline & & & Nybelinia goreensis & $\mathrm{NC}$ \\
\hline & & & Nybelinia sp. B & $\mathrm{NC}$ \\
\hline & \multirow[t]{6}{*}{ Nemipteridae } & \multirow[t]{6}{*}{ Nemipterus furcosus (Valenciennes, 1830) } & Callitetrarhynchus gracilis & $\mathrm{NC}$ \\
\hline & & & Floriceps minacanthus & $\mathrm{NC}$ \\
\hline & & & Nybelinia indica & $\mathrm{NC}$ \\
\hline & & & Nybelinia goreensis & $\mathrm{NC}$ \\
\hline & & & Nybelinia queenslandensis & $\mathrm{NC}$ \\
\hline & & & $\begin{array}{l}\text { Symbothriorhynchus } \\
\text { tigaminacanthus }\end{array}$ & $\mathrm{NC}$ \\
\hline & Polynemidae & Polynemus heptadactyla (Cuvier, 1829) & Callitetrarhynchus gracilis & GBR \\
\hline & Pomacentridae & Abudefduf whitleyi Allen \& Robertson, 1974 & Callitetrarhynchus gracilis & GBR \\
\hline & \multirow[t]{3}{*}{ Sciaenidae } & \multirow[t]{3}{*}{ Johnius borneensis (Bleeker, 1851) } & Callitetrarhynchus gracilis & GBR \\
\hline & & & Nybelinia strongyla & GBR \\
\hline & & & Proemotobothrium southwelli & GBR \\
\hline & \multirow[t]{9}{*}{ Scombridae } & Euthynnus affinis (Cantor, 1849) & Floriceps minacanthus & GBR \\
\hline & & Euthynnus alletteratus (Rafinesque, 1810) & Floriceps minacanthus & GBR \\
\hline & & $\begin{array}{l}\text { Grammatorcynus bicarinatus } \\
\quad \text { (Quoy \& Gaimard, 1825) }\end{array}$ & Floriceps minacanthus & GBR \\
\hline & & Sarda australis (Macleay, 1881) & Heteronybelinia estigmena & GBR \\
\hline & & Scomberomorus commerson (Lacépède, 1800) & Callitetrarhynchus gracilis & GBR, NC \\
\hline & & & Grillotiella exile & GBR \\
\hline & & & Pterobothrium acanthotruncatum & GBR \\
\hline & & Scomberomorus queenslandicus Munro, 1943 & Callitetrarhynchus gracilis & GBR \\
\hline & & Thunnus obesus (Lowe, 1839) & Hepatoxylon trichiuri & $\mathrm{NC}$ \\
\hline & \multirow[t]{29}{*}{ Serranidae } & \multirow{2}{*}{ Cephalopholis boenak (Bloch, 1790) } & Callitetrarhynchus gracilis & $\mathrm{NC}$ \\
\hline & & & Floriceps minacanthus & GBR \\
\hline & & \multirow[t]{4}{*}{ Cephalopholis cyanostigma (Valenciennes, 1828) } & Pseudolacistorhynchus shipleyi & GBR \\
\hline & & & Callitetrarhynchus gracilis & GBR \\
\hline & & & Floriceps minacanthus & GBR \\
\hline & & & Pseudotobothrium dipsacum & GBR \\
\hline & & \multirow[t]{4}{*}{ Cephalopholis miniata (Forsskål, 1775) } & Callitetrarhynchus gracilis & GBR \\
\hline & & & Floriceps minacanthus & GBR, NC \\
\hline & & & Pseudolacistorhynchus heroniensis & GBR \\
\hline & & & Pseudotobothrium dipsacum & $\mathrm{NC}$ \\
\hline & & \multirow[t]{3}{*}{ Cephalopholis sonnerati (Valenciennes, 1828) } & Floriceps minacanthus & $\mathrm{NC}$ \\
\hline & & & Pseudolacistorhynchus heroniensis & $\mathrm{NC}$ \\
\hline & & & Pseudotobothrium dipsacum & $\mathrm{NC}$ \\
\hline & & Cephalopholis spiloparaea (Valenciennes, 1828) & Callitetrarhynchus gracilis & $\mathrm{NC}$ \\
\hline & & \multirow[t]{3}{*}{ Cephalopholis urodeta (Schneider, 1801) } & Floriceps minacanthus & $\mathrm{NC}$ \\
\hline & & & Pseudolacistorhynchus shipleyi & $\mathrm{NC}$ \\
\hline & & & Pseudotobothrium dipsacum & $\mathrm{NC}$ \\
\hline & & Cromileptes altivelis (Valenciennes, 1828) & Callitetrarhynchus gracilis & GBR \\
\hline & & \multirow[t]{5}{*}{ Epinephelus coioides (Hamilton, 1822) } & Dasyrhynchus pacificus & $\mathrm{NC}$ \\
\hline & & & Floriceps minacanthus & $\mathrm{NC}$ \\
\hline & & & Pseudogilquinia pillersi & $\mathrm{NC}$ \\
\hline & & & Pseudolacistorhynchus heroniensis & $\mathrm{NC}$ \\
\hline & & & Pseudotobothrium dipsacum & GBR, NC \\
\hline & & \multirow[t]{4}{*}{ Epinephelus chlorostigma (Valenciennes, 1828) } & Callitetrarhynchus gracilis & $\mathrm{NC}$ \\
\hline & & & Dasyrhynchus pacificus & $\mathrm{NC}$ \\
\hline & & & Diesingium cf lomentaceum & $\mathrm{NC}$ \\
\hline & & & Pseudolacistorhynchus heroniensis & $\mathrm{NC}$ \\
\hline & & \multirow[t]{2}{*}{ Epinephelus cyanopodus (Richardson, 1846) } & Floriceps minacanthus & $\mathrm{NC}$ \\
\hline & & & Pseudolacistorhynchus heroniensis & $\mathrm{NC}$ \\
\hline
\end{tabular}


Table 2. (continued)

\begin{tabular}{|c|c|c|c|c|}
\hline Order & Family & Host species & Parasites & Location \\
\hline & & \multirow[t]{2}{*}{ Epinephelus fasciatus (Forsskål, 1775) } & Callitetrarhynchus gracilis & $\mathrm{NC}$ \\
\hline & & & Pseudolacistorhynchus heroniensis & GBR, NC \\
\hline & & Epinephelus howlandi (Günther, 1873) & Pseudolacistorhynchus heroniensis & $\mathrm{NC}$ \\
\hline & & \multirow[t]{2}{*}{ Epinephelus maculatus (Bloch, 1790) } & Floriceps minacanthus & $\mathrm{NC}$ \\
\hline & & & Otobothrium parvum & $\mathrm{NC}$ \\
\hline & & Epinephelus malabaricus (Bloch \& Schneider, 1801) & Pseudotobothrium dipsacum & $\mathrm{NC}$ \\
\hline & & \multirow[t]{2}{*}{ Epinephelus ongus Bloch, 1793} & Pseudolacistorhynchus heroniensis & GBR \\
\hline & & & Pseudolacistorhynchus shipleyi & $\mathrm{GBR}, \mathrm{NC}$ \\
\hline & & \multirow[t]{2}{*}{ Epinephelus polyphekadion (Bleeker, 1849) } & Pseudolacistorhynchus heroniensis & $\mathrm{NC}$ \\
\hline & & & Pseudolacistorhynchus shipleyi & $\mathrm{NC}$ \\
\hline & & \multirow[t]{2}{*}{ Epinephelus retouti (Bleeker, 1868) } & Pseudotobothrium dipsacum & $\mathrm{NC}$ \\
\hline & & & Callitetrarhynchus gracilis & $\mathrm{NC}$ \\
\hline & & Epinephelus quoyanus (Valenciennes, 1830) & Pseudolacistorhynchus heroniensis & GBR \\
\hline & & \multirow[t]{2}{*}{ Epinephelus rivulatus (Valenciennes, 1830) } & Callitetrarhynchus gracilis & $\mathrm{NC}$ \\
\hline & & & Pseudolacistorhynchus heroniensis & $\mathrm{NC}$ \\
\hline & & Plectropomus areolatus (Rüppell, 1830) & Floriceps minacanthus & GBR \\
\hline & & \multirow[t]{3}{*}{ Plectropomus laevis (Lacépède, 1801) } & Floriceps minacanthus & $\mathrm{NC}$ \\
\hline & & & Pseudogilquinia pillersi & $\mathrm{NC}$ \\
\hline & & & Pseudotobothrium dipsacum & $\mathrm{NC}$ \\
\hline & & \multirow[t]{3}{*}{ Plectropomus leopardus (Lacépède, 1802) } & Floriceps minacanthus & GBR, NC \\
\hline & & & Pseudolacistorhynchus heroniensis & $\mathrm{GBR}, \mathrm{NC}$ \\
\hline & & & Pseudotobothrium dipsacum & GBR, NC \\
\hline & & \multirow[t]{3}{*}{ Plectropomus maculatus (Bloch, 1790) } & Callitetrarhynchus gracilis & GBR \\
\hline & & & Pseudotobothrium dipsacum & GBR \\
\hline & & & Pterobothrium acanthotruncatum & GBR \\
\hline & & \multirow[t]{2}{*}{ Variola louti (Forsskål, 1775) } & Callitetrarhynchus gracilis & $\mathrm{NC}$ \\
\hline & & & Floriceps minacanthus & $\mathrm{NC}$ \\
\hline & & \multirow{3}{*}{ Sphyraena jello Cuvier, 1829} & Bombycirhynchus sphaerenaicum & GBR \\
\hline & & & Floriceps minacanthus & GBR \\
\hline & & & Pterobothrium pearsoni & GBR \\
\hline & & Sphyraena obtusata Cuvier, 1829 & Callitetrarhynchus gracilis & GBR \\
\hline & & \multirow[t]{2}{*}{ Sphyraena putnamae Jordan \& Seale, 1905} & Floriceps minacanthus & $\mathrm{NC}$ \\
\hline & & & Heteronybelinia estigmena & $\mathrm{NC}$ \\
\hline \multirow[t]{2}{*}{ Syngnathiformes } & Fistulariidae & Fistularia commersonii Rüppell, 1838 & Dasyrhynchus basipunctatus & GBR \\
\hline & Trichiuridae & Trichiurus lepturus Linnaeus, 1758 & Heteronybelinia estigmena & $\mathrm{NC}$ \\
\hline \multirow[t]{20}{*}{ Tetraodontiformes } & Balistidae & Abalistes filamentosus Matsuura \& Yoshino, 2004 & Dasyrhynchus basipunctatus & $\mathrm{NC}$ \\
\hline & & & Pseudolacistorhynchus heroniensis & $\mathrm{NC}$ \\
\hline & & & Pseudotobothrium dipsacum & $\mathrm{NC}$ \\
\hline & & Abalistes stellatus (Anonymous, 1798) & Dasyrhynchus basipunctatus & $\mathrm{NC}$ \\
\hline & & & Pseudolacistorhynchus heroniensis & $\mathrm{NC}$ \\
\hline & & & Pseudotobothrium dipsacum & GBR, NC \\
\hline & & Pseudobalistes fuscus (Bloch \& Schneider, 1801) & Dasyrhynchus basipunctatus & $\mathrm{NC}$ \\
\hline & & & Pseudolacistorhynchus heroniensis & $\mathrm{NC}$ \\
\hline & & & Pseudotobothrium dipsacum & $\mathrm{NC}$ \\
\hline & & Rhinecanthus aculeatus (Linnaeus, 1758) & Pseudolacistorhynchus shipleyi & GBR \\
\hline & & & Pseudotobothrium dipsacum & GBR \\
\hline & & Rhinecanthus rectangulus (Bloch \& Schneider, 1801) & Pseudotobothrium dipsacum & GBR \\
\hline & & Sufflamen fraenatus (Latreille, 1804) & Heteronybelinia sp. C & $\mathrm{NC}$ \\
\hline & & & Pseudolacistorhynchus shipleyi & GBR, NC \\
\hline & Diodontidae & Diodon hystrix Linnaeus, 1758 & Floriceps saccatus & GBR, NC \\
\hline & & & Hepatoxylon trichiuri & GBR, NC \\
\hline & & & Molicola horridus & GBR, NC \\
\hline & & & Nybelinia indica & $\mathrm{GBR}, \mathrm{NC}$ \\
\hline & & Diodon liturosus Shaw, 1804 & Floriceps saccatus & GBR \\
\hline & & & Molicola horridus & GBR \\
\hline
\end{tabular}


Table 2. (continued)

\begin{tabular}{|c|c|c|c|c|}
\hline Order & Family & Host species & Parasites & Location \\
\hline \multirow{8}{*}{ Scorpaeniformes } & \multirow{4}{*}{ Tetraodontidae } & \multirow{3}{*}{ Lagocephalus sceleratus (Gmelin, 1789) } & Dasyrhynchus basipunctatus & $\overline{\mathrm{NC}}$ \\
\hline & & & Nybelinia indica & $\mathrm{NC}$ \\
\hline & & & Dasyrhynchus basipunctatus & $\mathrm{NC}$ \\
\hline & & \multirow[t]{2}{*}{ Triodon macropterus Lesson, 1831} & Callitetrarhynchus gracilis & $\mathrm{NC}$ \\
\hline & \multirow{3}{*}{ Platycephalidae } & & Dasyrhynchus basipunctatus & $\mathrm{NC}$ \\
\hline & & \multirow[t]{2}{*}{ Cymbacephalus beauforti (Knapp, 1973) } & Callitetrarhynchus speciosus & $\mathrm{NC}$ \\
\hline & & & Pseudotobothrium dipsacum & $\mathrm{NC}$ \\
\hline & Scorpaenidae & Dendrochirus zebra (Cuvier, 1829) & Nybelinia aequidentata & $\mathrm{NC}$ \\
\hline
\end{tabular}

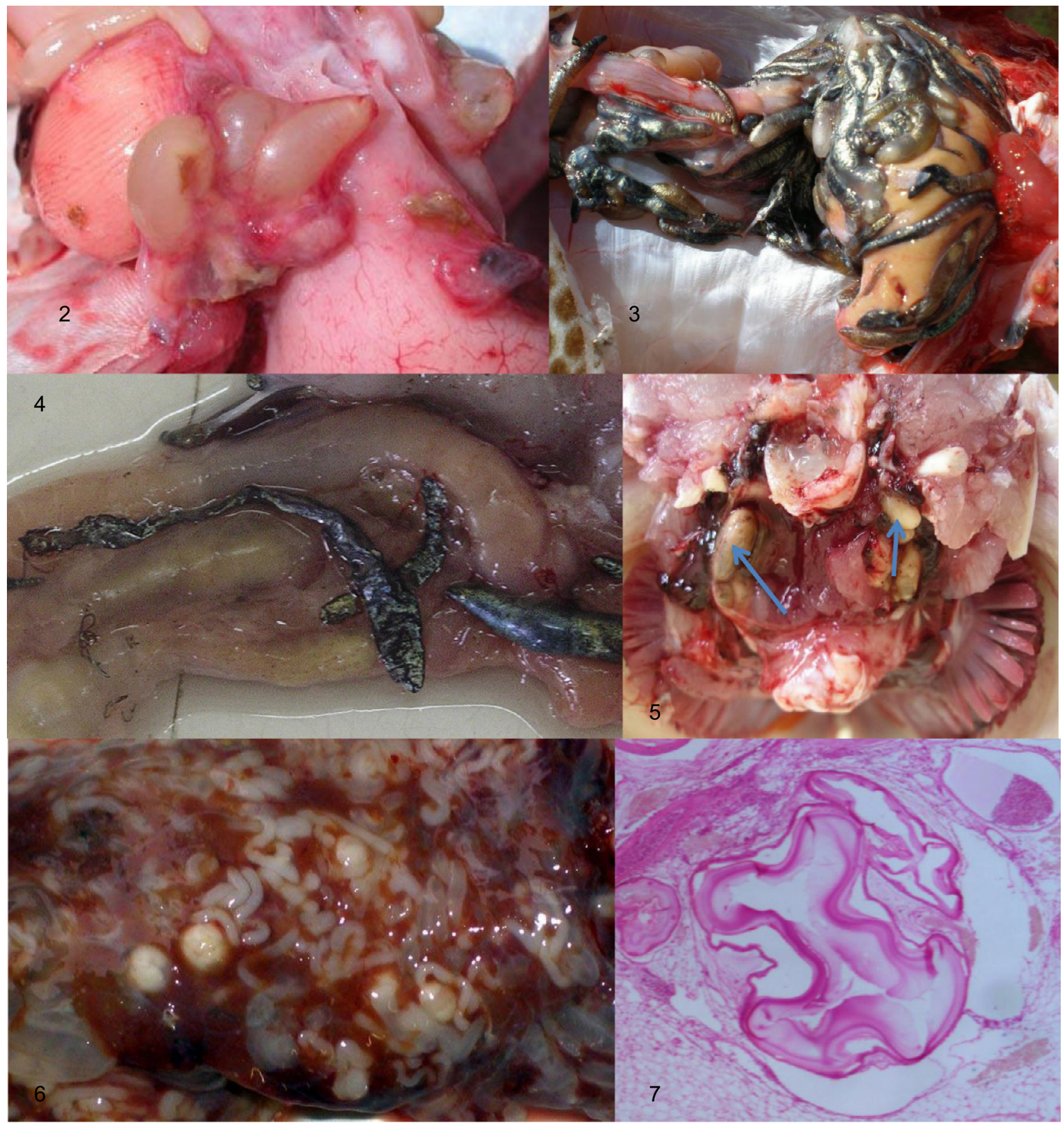

Figures 2-7. Metacestodes of trypanorhynch cestodes from teleost fishes. 2. Viable plerocerci of Callitetrarhynchus gracilis in the body cavity of Scomberomorus commerson. 3. Melanised trypanorhynch plerocerci in the body cavity of Epinephelus sp. 4. Melanised and contracted cysts of trypanorhynch metacestodes in the body cavity of Cephalopholis miniata; no viable plerocerci were recovered from these cysts. 5. Plerocerci of Pseudogilquinia spp. (arrows) around the oesophagus of Lethrinus nebulosus. 6. Merocerci of Molicola horridus in the liver of Diodon hystrix. 7. Plerocerci of Grillotiella exile in the gill arches of Scomberomorus commerson (histological section). 
Table 3. Summary of the fully identified taxa of larval trypanorhynch cestodes found in teleost fishes from the Great Barrier Reef and from New Caledonia.

\begin{tabular}{lcccc}
\hline Order & Total number of species & Great Barrier Reef & New Caledonia & Number of shared species $(\%)$ \\
\hline Gymnorhynchoidea & 5 & 5 & 1 & $1(20 \%)$ \\
Lacistorhynchoidea & 14 & 10 & 12 & $8(57 \%)$ \\
Otobothrioidea & 6 & 3 & 5 & $2(33 \%)$ \\
Tentacularioidea & 9 & 6 & 7 & $5(55 \%)$ \\
All orders & 33 & 22 & 23 & $15(45 \%)$ \\
\hline
\end{tabular}

plerocercoids most closely resemble $N$. lingualis but cannot be assigned to this species with certainty.

\section{Nybelinia sp. B (Fig. 9)}

Material examined: plerocercoid from Parupeneus multifasciatus (Quoy \& Gaimard, 1825), New Caledonia, MNHN JNC2172 C4.

Scolex length 1750, pars bothrialis 1100, pars vaginalis 1000, bulbs elongate, 560 long, velum 200, metabasal hooks: length 20, base 14 .

Remarks

This specimen most closely resembles $N$. strongyla Dollfus, 1960 in scolex length, bulb length and hook size and shape, but differs in the length of the velum (690-830 in $N$. strongyla compared with 200 in the present material).

\section{Heteronybelinia sp. C (Fig. 10)}

Material examined: plerocercoid from Sufflamen fraenatus (Latreille, 1804), New Caledonia, MNHN JNC3034.

Scolex length 1440, pars bothrialis 770, pars vaginalis 680 , bulbs elongate, bulb length 375 , velum 125 , metabasal hooks on antibothrial surface: length 17-19, base 8; on bothrial surface: length 25 , base 18; basal armature heteromorphous.

Remarks

This specimen clearly belongs to Heteronybelinia as the hooks differ markedly in shape on the bothrial versus the antibothrial surfaces of the tentacle. Hook sizes are closest to H. eureia (Dollfus, 1960), but the specimen differs from this species in the number of hooks per half spiral and by the fact that in this specimen the bulbs are entirely posterior to the pars bothrialis while in H. eureia, they do not extend beyond the pars bothrialis. Therefore, this specimen cannot be accommodated within any known species of Heteronybelinia.

4. Nybelinia basimegacantha Carvajal, Campbell \& Cornford, 1976 (Fig. 11)

Material examined: plerocercoid from Parupeneus multifasciatus (Quoy \& Gaimard, 1825), New Caledonia, MNHN JNC2111 C1; plerocercoid from Neoniphon sammara (Forsskål, 1775), New Caledonia, MNHN JNC2552.

Specimen from P. multifasciatus: Scolex length 2600, pars bothrialis 1400 , pars vaginalis 900 , bulb length 1060 , bulb width 130, velum 90 .

Specimen from N. sammara: Scolex length 1380, pars bothrialis 840 , pars vaginalis 350 ; bulb length 450 , bulb width 70 , velum 70 .

\section{Remarks}

Two specimens have been identified as belonging to this species with its characteristic armature. In spite of the fact that the armature of both specimens is identical, scolex measurements differed substantially and for this reason, the measurements of both specimens are presented. The specimen from P. multifasciatus although quite flattened, corresponds more closely with the original description of the species, also from P. multifasciatus from Hawaii [10]. In the specimen from N. sammara, all measurements are shorter but the tentacular armature is identical.

\section{Discussion}

\section{General comments}

Although the records of trypanorhynch infections listed here are based on the dissection of thousands of fish from both the GBR and NC, the data collected are based on opportunistic collecting and must be viewed in this light. Few prevalence or intensity data were collected and the data are based largely on the presence of trypanorhynch metacestodes. Fish examined that did not harbour metacestodes were not included in the data presented in the tables but representative examples have been indicated in the results.

In spite of these limitations, the large numbers of metacestodes collected from both regions provide a significant basis for comparing trypanorhynch metacestodes of teleosts inhabiting coral reefs.

Several features are evident from the data presented. In spite of potential differences in the fish faunas between the two regions examined and possible biases in sampling approaches, an extremely large number of fish specimens (thousands) was examined at each locality and even though the methods of examination varied to some degree, the study encompassed a wide range of fish families at both sites. Overall, $45 \%$ of the trypanorhynch species recorded here occurred in both regions. In addition, the trypanorhynch species most commonly encountered were similar in both locations. Records of adults from elasmobranchs from both of these regions provided additional information on potential life cycles and the collection included numerous new host and geographical records.

\section{Host specificity}

Notwithstanding the opportunistic nature of the collecting, several aspects of host specificity are detectable within the data set and are worthy of discussion particularly since Palm \& Caira, 2008 [30] have shown that specificity of the larval stages of trypanorhynchs is generally lower than that of the adults. First, it is evident that several fish taxa were rarely infected with trypanorhynchs. Thus, despite examination of substantial 

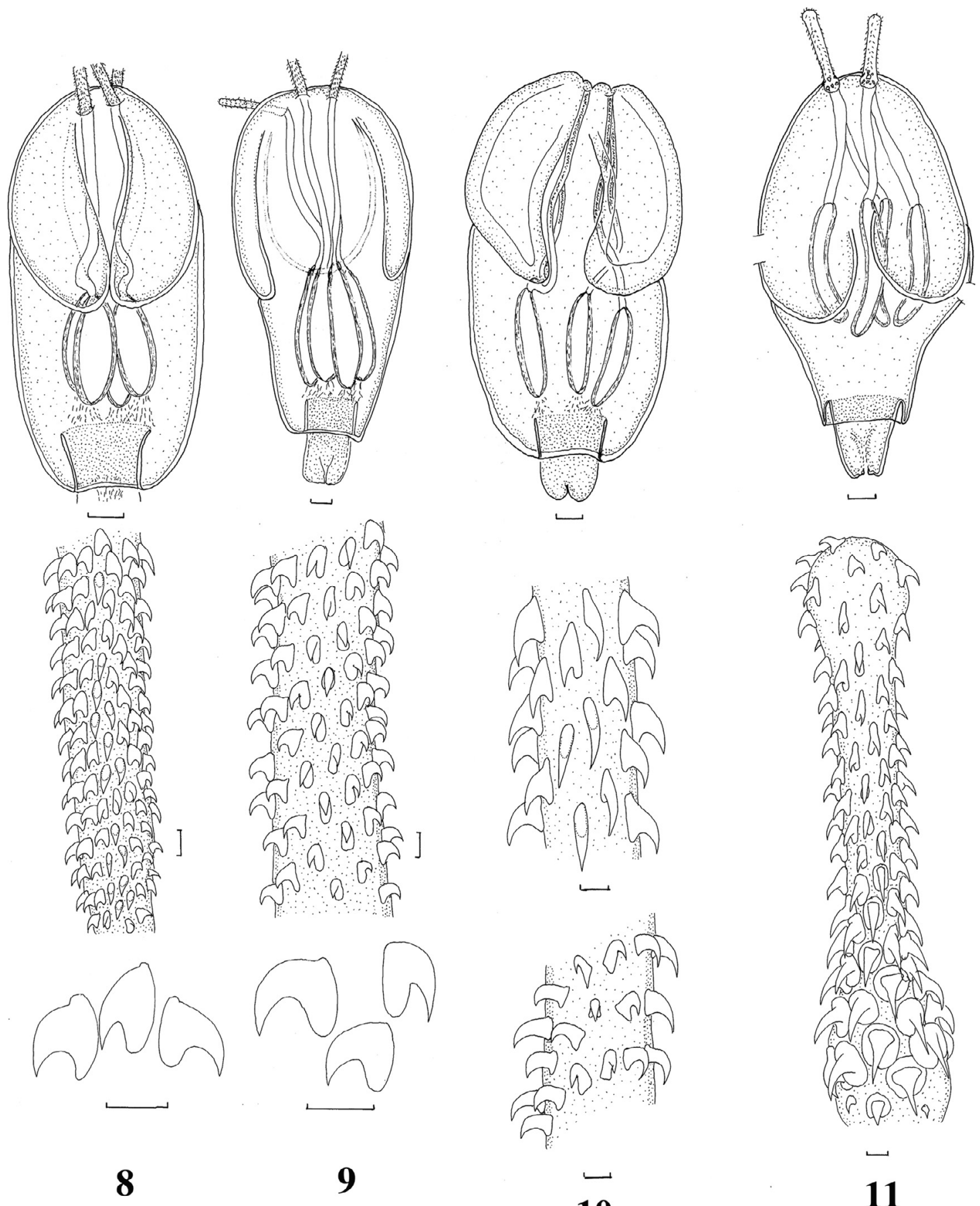

9

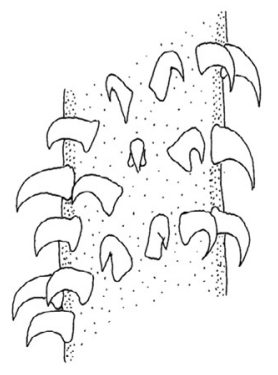

10

11

Figures 8-11. Tentacularioid metacestodes incompletely identified. 8. Nybelinia sp. A from Herklotsichthys quadrimaculatus (Rüppell, 1937). Scolex, basal and metabasal armature, hook profiles. Scale-bars: scolex and tentacle, $0.1 \mathrm{~mm}$; hooks, $0.01 \mathrm{~mm}$. 9. Nybelinia sp. B from Parupeneus multifasciatus (Quoy \& Gaimard, 1825). Scolex, basal and metabasal armature, hook profiles. Scale-bars: scolex and tentacle, $0.1 \mathrm{~mm}$; hooks, 0.01 $\mathrm{mm}$. 10. Heteronybelinia sp. C from Sufflamen fraenatus (Latreille, 1804). Scolex, bothrial metabasal armature and antibothrial metabasal armature. Scale-bars: scolex $0.1 \mathrm{~mm}$; hooks $0.01 \mathrm{~mm}$. 11. Nybelinia basimegacantha Carvajal, Campbell \& Cornford, 1976, specimen from Neoniphon sammara (Forsskål, 1775). Scolex, basal and metabasal armature. Scale-bars: scolex $0.1 \mathrm{~mm}$; tentacle $0.01 \mathrm{~mm}$.

numbers of Blenniidae, Chaetodontidae, Gobiidae, Kyphosidae and Scaridae, no trypanorhynchs were found in these taxa. Other taxa strikingly underrepresented, though heavily sampled, were the Acanthuridae, Pomacentridae and Echeneidae. We do not suggest that these taxa have been exhaustively examined, but certainly they are depauperate relative to families such as the Balistidae, Lethrinidae, Scombridae and Serranidae.

Among the teleost fishes that were infected, there was evidence of both stenoxenicity (parasitism of closely related 
species) and euryxenicity (parasitism of distantly or ecologically related species). In the stenoxenous category, Molicola horridus was seen in two species of Diodontidae, Pterobothrium australiense has been seen only in labrids (one record), Pseudogilquinia microbothria was found only in lethrinids (both in NC and the GBR) and Dasyrhynchus basipunctatus occurred overwhelmingly in tetraodontiforms (five species) although also once in a fistulariid. The apparently restricted distributions of such species are doubtless subject to refinement with further collecting but it seems highly unlikely that they will prove to be euryxenous in the same way as are some other species.

We detected some evidence of the absence of trypanorhynch species in particular fish groups. The best evidence comes from the family Serranidae which is probably the most thoroughly characterised for its trypanorhynch fauna. The serranid fishes collected tend to be large and easily examined for trypanorhynchs with which they are often heavily infected. Our results incorporate reports from 25 serranid species and of the 181 host/parasite combinations detected, 55 were from serranids; the next highest number of combinations came from the Lethrinidae with 14 . The extent to which the characterisation of this family is comprehensive is demonstrated by the fact that six of the ten trypanorhynch species recorded in this family have been reported from more than one serranid species; three species were found in ten or more serranid species although four species were found in only one. We infer that the true trypanorhynch richness is thus not likely to be very much greater than the 10 species reported so far in this region. Thus, we predict that species that have been reported relatively frequently in other fishes are genuinely absent, rather than have simply not yet been collected. Most striking in this respect are the species of the Tentacularioidea. Twelve species of this superfamily are reported here in 34 host/parasite combinations, but none in serranids. The apparent absence of a range of species from the Serranidae thus appears consistent with the high host specificity seen for the species described above.

Several species showed remarkably low specificity. Thus, Callitetrarhynchus gracilis was reported here from five fish orders and 18 families, Floriceps minacanthus from two orders and six families, Pseudotobothrium dipsacum from three orders and six families, Pseudolacistorhynchus heroniensis from two orders and four families and Pseudolacistorhynchus shipleyi from three orders and five families. The absence of any detectable specificity in these species leads to the prediction that further sampling will lead to even larger host ranges for these species.

Callitetrarhynchus gracilis exhibited the widest host range and has a cosmopolitan distribution [27] with carcharhinid sharks as its primary definitive hosts in the Australian region [1]. Currently recorded in the intermediate stage from approximately 130 species of teleosts [16, 27, 29], 23 new host records have been added in the present study.

Floriceps minacanthus appears to be limited to the IndoPacific region, and again, its known definitive hosts are carcharhinid sharks [26], with adults having been reported from four species of Carcharhinus. However, the present record in Triaenodon obesus is the first from a shark not belonging to this genus. Plerocerci have been reported from 13 species of teleosts [27, 29] from the Red Sea, Australia and off Indonesia and Hawaii while 14 new species of teleosts are reported here as hosts.

Pseudotobothrium dipsacum was also found in a wide variety of teleosts. It has previously been reported from numerous species of teleosts ranging from the west coast of Africa to Australia $[4,27]$. Eight new hosts, all from New Caledonia, have been added in the present study. In spite of its wide host range and distribution, its definitive hosts remain unknown.

Pseudolacistorhynchus heroniensis is known only from the GBR and from NC but is found in a wide range of teleosts, with 12 new teleost hosts being added in the current study. The only record of the adult parasite is a single collection from Stegostoma fasciatum from New Caledonia [6]. The specimens collected were either immature or hyperapolytic such that some doubt exists as to whether this is the usual definitive host species.

Pseudolacistorhynchus shipleyi occurs widely in the Indo-West Pacific, with the adults being found in Nebrius ferrugineus off Sri Lanka [2]. In the current study, eleven new intermediate host records are reported.

The above five species occurred in a wide variety of teleost hosts with serranids (25 species), carangids (5), balistids (5), scombrids (5) and sphyraenids (5) being most frequently encountered. The same five species of trypanorhynch were the most commonly encountered species both on the Great Barrier Reef and off New Caledonia in spite of obvious differences in the species of fish infected at the two localities. There was no intentional bias in collecting activities, but it may have been that more of these larger fishes were collected than other smaller taxa.

Other species of trypanorhynch had a more restricted host distribution. Limited data on prevalence based on a single series of collections from Lizard Island suggested that trypanorhynch larvae were prevalent in larger fishes (serranids, sphyraenids, scombrids, lutjanids) but in small fish (a single family, Apogonidae) they occurred at a very low prevalence. However, these data were based on a very small sample of fish and need to be interpreted with caution.

Overall, the patterns of host specificity seen here, a mixture of stenoxenicity and euryxenicity, resemble that reported by Chambers et al., 2000 [11] for tetraphyllidean (sensu lato) metacestodes of GBR fishes. In that study, metacestode Type 4 was found in two orders and 12 families, whereas Types 9 and 10 were found only in labrids. However, in the study of tetraphyllidean metacestodes it is often not possible to be confident that a single morphotype represents only one species whereas the complex morphology of trypanorhynch scoleces makes identification to species quite reliable.

\section{Biogeography}

Of the 33 trypanorhynch species reported here, 15 (45\%) were found both in NC and on the GBR. Almost certainly this number underestimates the level of sharing between the two areas. Noticeably, the nine species reported in the largest number of host/parasite combinations were all found at both sites. Of the 21 species found in only one or two host/parasite combinations, only one (Molicola horridus) was found both in NC and on the 
GBR. It seems likely, or at least possible, that some species are restricted to one or other of the two sites but at present the evidence is generally marginal in this respect. The only robust parasitological study of which we are aware that has previously compared parasites of $\mathrm{NC}$ and the GBR is that of McNamara et al., 2012 [26] who analysed monorchiid trematodes of chaetodontids from NC and the GBR (as well as other sites in the Tropical Indo-West Pacific [TIWP]). Thirteen species of Hurleytrematoides Yamaguti, 1953 were found in total for the two sites of which just six were found at both sites for a similarity of $46 \%$; four species were found only from the GBR and three only from NC. In every case, hosts suitable for the species not found in each area had been examined in numbers sufficient to suggest that they would have been found if present. The proportion of monorchiid species shared (46\%) is thus remarkably similar to that for the trypanorhynchs. Given the much stricter specificity of monorchiids of chaetodontids (none known convincingly other than from chaetodontids) than of trypanorhynch metacestodes in general, we predict that further sampling for trypanorhynchs will see the levels of sharing increase.

Of the species found, eight (C. gracilis, F. saccatus, $G r$. exile, Hep trichiuri, Het. estigmena, M. horridus, $N$. goreensis, $O$. penetrans) have a cosmopolitan distribution, based on records in Palm, 2004 [27], while ten species are widely distributed in the Tropical Indo-West Pacific (TIWP) (D. pacifcus, F. minacanthus, N. basimegacantha, N. indica, Psgi. microbothria, Psgi. pillersi, D. basipunctatus, Psl. shipleyi, Psd. dipsacum, Pt. acanthotruncatum). By contrast, seven species occur only in south-east Asia and Australasia ( N. queenslandensis, O. alexanderi, O. parvum, Psl. heroniensis, Psl. nanus, Pt. australiense, S. tigaminacanthus). Several additional species (e.g. Pt. lintoni) with few, highly disjunct records are difficult to categorise. Nevertheless, with many of the trypanorhynch species encountered having extremely wide geographical distributions [31], it was not surprising that the species found on the GBR and from NC were broadly similar.

\section{Localisation in host}

Apart from potential differences in the species of fish present at the two sites studied, or their abundance and hence ease of obtaining a particular species, other factors may be involved such as the location of trypanorhynch metacestodes in the body of the teleost. Most are found in the body cavity and are easily recognised. However, the metacestodes of Gr. exile occur only in the gill arches of Sc. commerson [35] and this site is not always examined for the presence of metacestodes. Similarly, the metacestodes of Psg. microbothria cluster around the oesophagus of L. nebulosus (unpublished) while those of $P t$. lintoni are found in the musculature (unpublished). Failure to examine sites other than the body cavities may lead to differences in the species recovered.

\section{Life cycles}

Combining the data obtained here with that available for adult trypanorhynchs in elasmobranchs in the same region has provided some insights into life cycles such as finding the adult of Pt. acanthotruncatum for the first time in Pristis zijsron. In addition, the definitive host range of F. minacanthus is expanded to include the shark Triaenodon obesus. Many life cycles remain to be identified, but broad scale collecting, such as that undertaken in this study, can be useful in identifying both potential intermediate and definitive hosts.

Species of Diodon warrant a particular mention as they are parasitised by several well-recognised trypanorhynch species including Floriceps saccatus and Molicola horridus. Infections with the latter species are particularly striking as much of the hepatic parenchyma may be replaced by metacestodes (Fig. 5). Species of Diodon are not only highly toxic [36], but can also inflate their bodies when threatened. As adults of these cestodes are found in large sharks such as Prionace glauca (Linnaeus, 1758) (see Dollfus, 1942) [14], it is tempting to assume that only large sharks are able to consume species of Diodon. Alternatively, it may be that the life cycles of these cestodes are completed using alternative intermediate hosts and their presence in species of Diodon indicates an occurrence in "dead-end" hosts. By comparison, in a study of the larval anisakid nematodes of teleosts off Lizard Island, Jabbar et al., 2012 [18] found no larval anisakids in their sample of tetraodontiform fishes, which would potentially be "deadend" hosts for these nematodes.

\section{Conclusion}

This is the first study to attempt to examine the trypanorhynch larval cestode fauna of coral reef teleosts in the west Pacific, examining reefs on the GBR and NC. The trypanorhynch fauna was dominated numerically by a small number of species at both sites with considerable similarity between the two localities examined. Although large numbers of teleosts were examined at both sites, it is most unlikely that the trypanorhynch fauna has been exhaustively surveyed and more detailed comparisons must await much more extensive sampling. Nevertheless, apart from characterising the general features of the fauna, this study has provided additional insights into host specificity and life cycles of these cestode parasites.

\section{Conflict of Interest}

The Editor-in-Chief of Parasite is one of the authors of this manuscript. COPE (Committee on Publication Ethics, http:// publicationethics.org/), to which Parasite adheres, advises special treatment in these cases. COPE wrote: "Editors should not be denied the ability to publish in their own journal, but they must not exploit their position. The journal must have a procedure for handling submissions from the editor or members of the editorial board that ensures that peer review is handled independently of the author/editor. This process should be detailed once the paper is published." In this case the peerreview process was handled by Invited Editor Dominique Vuitton.

Acknowledgements. Collecting on the GBR was funded by the Australian Research Council and the Australian Biological Resources Study, the latter in part through the CReefs Program. 
In New Caledonia, the following students of JLJ participated in the parasitological survey: Julie Mounier, Charles Beaufrère, Anaïs Guillou, Audrey Guérin, Damien Hinsinger, Éric Bureau, Chloé Journo, Violette Justine, Amandine Marie, Aude Sigura, Sophie Olivier, Guilhem Rascalou, Géraldine Colli, Lenaïg Hemery, Pierpaolo Brena, Cyndie Dupoux, Isabelle Mary, Adeline Grugeaud, Marine Briand and Charlotte Schoelinck. Claude Chauvet (UNC, Nouméa) provided several fishes. Angelo di Matteo (IRD) provided technical help. Visiting colleagues who participated were Ian Beveridge, Louis Euzet, Eva Řehulková, František Moravec, Milan Gelnar, Bernard Marchand and Susan Lim. Certain fishes from New Caledonia were identified (often from photographs) by Jack Randall (Bishop Museum, Hawaii), Ronald Fricke (Staatliches Museum für Naturkunde, Stuttgart, Germany), Kent E. Carpenter (Old Dominion University, Norfolk, Virginia, USA), Philippe Borsa (IRD, Nouméa), Bernard Séret (IRD and MNHN, Paris) and Samuel Iglésias (MNHN, Paris).

\section{References}

1. Beveridge I, Campbell RA. 1996. New records and redescriptions of trypanorhynch cestodes from Australian fishes. Records of the South Australian Museum, 29, 1-22.

2. Beveridge I, Campbell RA. 1998. Re-examination of the trypanorhynch cestode collections of A.E. Shipley, J. Hornell and T. Southwell with the erection of a new genus, Trygonicola, and redescriptions of seven species. Systematic Parasitology, $39,1-34$.

3. Beveridge I, Campbell RA. 2001. Proemotobothrium n.g. (Cestoda: Trypanorhyncha), with the redescription of $P$. linstowi (Southwell, 1912) n. comb. and description of P. southwelli n. sp. Systematic Parasitology, 48, 223-233.

4. Beveridge I, Campbell RA, Jones MK. 2000. New records of the cestode genus Pseudotobothrium (Trypanorhyncha: Otobothriidae) from Australian fishes. Transactions of the Royal Society of South Australia, 124, 151-162.

5. Beveridge I, Chauvet C, Justine J-L. 2007. Redescription of Pseudogilquinia pillersi (Southwell, 1929) (Cestoda: Trypanorhyncha) from serranid and lethrinid fishes from New Caledonia and Australia. Acta Parasitologica, 52, 213-218.

6. Beveridge I, Justine J-L. 2007. Pseudolacistorhynchus nanus n. sp. (Cestoda: Trypanorhyncha) parasitic in the spiral valve of the zebra shark, Stegostoma fasciatum (Hermann, 1783). Transactions of the Royal Society of South Australia, 132, 177-183.

7. Bouchet P, Lozouet P, Maestrati P, Heros V. 2002. Assessing the magnitude of species richness in tropical marine environments: exceptionally high numbers of molluses at a New Caledonia site. Biological Journal of the Linnean Society, London, 75, 421-436.

8. Campbell RA, Beveridge I. 1996. Revision of the family Pterobothriidae Pintner, 1931 (Cestoda: Trypanorhyncha). Invertebrate Taxonomy, 10, 617-662.

9. Carpenter KE, Abrar M, Aeby G, Aronson RB, Banks S, Bruckner A, Chiriboga A, Cortés J, Delbeek JC, DeVantier L, Edgar GJ, Edwards AJ, Fenner D, Guzmán HM, Hoeksema AW, Hodgson G, Johan O, Licuanan WY, Livingstone SR, Lovell ER, Moore JA, Obura DO, Ochavillo D, Polidoro EA, Precht WF, Quibilan MC, Reboton C, Richards ZT, Rogers AD, Sanciangco J, Sheppard A, Sheppard C, Smith J, Stuart S, Turak E, Veron JEN, Wallace C, Weil E, Wood E. 2008. One third of reef building corals face elevated extinction risks from climate change and local effects. Science, 321, 560-563.

10. Carvajal JG, Campbell RA, Cornford EM. 1976. Some trypanorhynch cestodes from Hawaiian fishes with the description of four new species. Journal of Parasitology, 62, 70-77.

11. Chambers CB, Cribb TH, Jones MK. 2000. Tetraphyllidean metacestodes of teleosts of the Great Barrier Reef, and the use of in vitro cultivation to identify them. Folia Parasitologica, 47, 285-292.

12. Chervy L. 2002. The terminology of larval cestodes or metacestodes. Systematic Parasitology, 52, 1-33.

13. Cribb TH, Bray RA, Barker SC, Adlard RD, Anderson GR. 1994. Ecology and diversity of digenean trematodes of reef and inshore fishes of Queensland. International Journal for Parasitology, 24, 851-960.

14. Dollfus RP. 1942. Études critiques sur les Tétrarhynques du Muséum de Paris. Archives du Muséum National d'Histoire Naturelle, Paris, 19, 1-466.

15. Froese R, Pauly D. 2014. FishBase, World Wide Web electronic publication. www.fishbase.org.

16. Haseli M, Malek M, Valinasab T, Palm HW. 2011. Trypanorhynch cestodes of teleost fish from the Persian Gulf, Iran. Journal of Helminthology, 85, 215-224.

17. Hoeg-Guldberg O, Bruno JF. 2010. The impact of climate change on the world's ecosystems. Science, 328, 1523-1528.

18. Jabbar A, Asnoussi A, Norbury LJ, Eisenbarth A, Shamsi S, Gasser RB, Lopata AL, Beveridge I. 2012. Larval anisakid nematodes in teleost fishes from Lizard Island, northern Great Barrier Reef, Australia. Marine and Freshwater Research, 63, 1283-1299.

19. Jensen K. 2009. Cestoda (Platyhelminthes) of the Gulf of Mexico, in Felder DL, Camp DK, Editors. Gulf of Mexico. Origin, Waters, and Biota. Texas A \& M University Press: USA, p. 487-522.

20. Justine J-L. 2010. Parasites of coral reef fish: how much do we know? With a bibliography of fish parasites in New Caledonia. Belgian Journal of Zoology, 140, 155-190.

21. Justine J-L, Beveridge I, Boxshall GA, Bray RA, Moravec F, Trilles J-P, Whittington ID. 2010. An annotated list of parasites (Isopoda, Copepoda, Monogenea, Digenea, Cestoda and Nematoda) collected from groupers (Serranidae, Epinephelinae) in New Caledonia emphasizes parasite biodiversity in coral reef fish. Folia Parasitologia, 54, 237-262.

22. Justine J-L, Beveridge I, Boxshall GA, Bray RA, Moravec F, Trilles J-P, Whittington ID. 2010. An annotated list of fish parasites (Copepoda, Monogenea, Digenea, Cestoda and Nematoda) collected from Emperors and Emperor Bream (Lethrinidae) in New Caledonia further highlights parasite biodiversity estimates on coral reef fish. Zootaxa, 2691, 1-40.

23. Justine J-L, Beveridge I, Boxshall GA, Bray RA, Miller TL, Moravec F, Trilles J-P, Whittington ID. 2012. An annotated list of fish parasites (Isopoda, Copepoda, Monogenea, Digenea, Cestoda, Nematoda) collected from Snappers and Bream (Lutjanidae, Nemipteridae, Caesionidae) in New Caledonia confirms high parasite biodiversity on coral reef fish. Aquatic Biosystems, 8, 22.

24. Lester RJG, Sewell KB. 1989. Checklist of parasites from Heron Island, Great Barrier Reef. Australian Journal of Zoology, 37, 101-128.

25. MacKenzie K. 1990. Cestode parasites as biological tags for mackerel (Scomber scombrus L.) in the northeast Atlantic. 
Jounal du Conseil International pour l'Exploration de la Mer, 46, 155-166.

26. McNamara MKA, Adlard RD, Bray RA, Sasal P, Cribb TH. 2012. Monorchiids (Platyhelminthes: Digenea) of chaetodontid fishes (Perciformes): biogeographical patterns in the tropical Indo-West Pacific. Parasitology International, 61, 288-306.

27. Palm HW. 2004. The Trypanorhyncha Diesing, 1863. PKSPLIPB Press: Bogor.

28. Palm HW, Beveridge I. 2002. Tentaculariid cestodes of the order Trypanorhyncha (Platyhelminthes) from the Australian region. Records of the South Australian Museum, 35, 49-78.

29. Palm HW, Bray RA. 2014. Marine Fish Parasitology in Hawaii. Westarp \& Partner: Hohenwarsleben.

30. Palm HW, Caira JN. 2008. Host specificity of adult versus larval cestodes of the elasmobranch tapeworm order Trypanorhyncha. International Journal for Parasitology, 38, 381-388.

31. Palm HW, Waeschenbach A, Littlewood DTJ. 2007. Genetic diversity in the trypanorhynch cestode Tentacularia coryphaenae Bosc, 1797: evidence for a cosmopolitan distribution and low host specificity in the teleost intermediate host. Parasitology Research, 101, 153-159.

32. Plaisance L, Caley MJ, Brainard RE, Knowlton N. 2011. The diversity of coral reefs: What are we missing? PLoS One, 6(10), e25026.

33. Rohde K. 1976. Marine parasitology in Australia. Search, 7, 477-482.

34. Sakanari J. 1989. Grillotia heroniensis, sp. nov., and G. overstreeti, sp. nov., (Cestoda: Trypanorhyncha) from Great Barrier Reef fishes. Australian Journal of Zoology, 37, 81-87.

35. Shaharom FM, Lester RJG. 1982. Description of and observations on Grillotia branchi n.sp., a larval trypanorhynch from the branchial arches of the Spanish mackerel, Scomberomorus commerson. Systematic Parasitology, 4, 1-6.

36. Trevett AJ, Mavo B, Warrell DA. 1997. Tetrodotoxic poisoning from ingestion of a porcupine fish (Diodon hystrix) in Papua New Guinea: nerve conduction studies. American Journal of Tropical Medicine and Hygiene, 56, 30-32.

Cite this article as: Beveridge I, Bray RA, Cribb TH \& Justine J-L: Diversity of trypanorhynch metacestodes in teleost fishes from coral reefs off eastern Australia and New Caledonia. Parasite, 2014, 21, 60.

\section{-0 PARASTE}

An international open-access, peer-reviewed, online journal publishing high quality papers on all aspects of human and animal parasitology

Reviews, articles and short notes may be submitted. Fields include, but are not limited to: general, medical and veterinary parasitology; morphology, including ultrastructure; parasite systematics, including entomology, acarology, helminthology and protistology, and molecular analyses; molecular biology and biochemistry; immunology of parasitic diseases; host-parasite relationships; ecology and life history of parasites; epidemiology; therapeutics; new diagnostic tools.

All papers in Parasite are published in English. Manuscripts should have a broad interest and must not have been published or submitted elsewhere. No limit is imposed on the length of manuscripts.

Parasite (open-access) continues Parasite (print and online editions, 1994-2012) and Annales de Parasitologie Humaine et Comparée (1923-1993) and is the official journal of the Société Française de Parasitologie. 